\title{
Ovule siRNAs methylate and silence protein-coding genes in trans
}

Diane Burgess ${ }^{1}$, Hiu Tung Chow ${ }^{2}$, Jeffrey W. Grover ${ }^{3}$, Michael Freeling ${ }^{1}$, Rebecca A. Mosher ${ }^{2}$

${ }^{1}$ Department of Plant and Microbial Biology, The University of California, Berkeley, CA 94720, USA

${ }^{2}$ The School of Plant Sciences, The University of Arizona, Tucson, AZ 85721, USA

${ }^{3}$ Department of Molecular \& Cellular Biology, The University of Arizona, Tucson, AZ 85721, USA; current address: Seven Bridges Genomics, Charlestown, MA 02129, USA

Corresponding author e-mail: rmosher@email.arizona.edu

\begin{abstract}
24-nt small interfering siRNAs maintain asymmetric DNA methylation at thousands of euchromatic transposable elements in plant genomes in a process call RNA-directed DNA Methylation (RdDM). Although this methylation occasionally causes transcriptional silencing of nearby protein-coding genes, direct targeting of methylation at coding sequences is rare. $\mathrm{RdDM}$ is dispensable for growth and development in Arabidopsis, but is required for reproduction in other plant species, such as Brassica rapa. 24-nt siRNAs are particularly abundant in reproductive tissue, due largely to overwhelming expression from a small number of loci in the ovule and developing seed coat, termed siren loci. Here we show that siRNAs are often produced from gene fragments embedded in siren loci, and that these siRNAs can trigger methylation in trans at related protein-coding genes. This trans-methylation is associated with transcriptional silencing of target genes and may be responsible for seed abortion in RdDM mutants. Furthermore, we demonstrate that a consensus sequence in at least two families of DNA transposons is associated with abundant siren expression, most likely through recruitment of the CLSY3 putative chromatin remodeller. This research describes a new mechanism whereby RdDM influences gene expression and sheds light on the role of RdDM during plant reproduction.
\end{abstract}

\section{INTRODUCTION}

In plants, DNA methylation can be initiated de novo via RNA-directed DNA Methylation (RdDM), however how and where RdDM is initiated often remains mysterious (Matzke and Mosher, 2014). RdDM begins when RNA Polymerase IV (Pol IV) and RNA-DEPENDENT RNA POLYERMASE 2 (RDR2) produce short non-coding double-stranded transcripts that are processed by DICER-LIKE 3 (DCL3) into 24-nt short interfering (si)RNAs (Blevins et al., 2015; Li et al., 2015; Singh et al., 2019; Zhai et al., 2015) One strand of these siRNA duplexes is then loaded into ARGONAUTE 4 (AGO4) or its relatives (Havecker et al., 2010). The AGO/siRNA complex interacts with non-coding transcripts produced by RNA Pol V and recruits DOMAINS REARRANGED METHYLTRANSFERASE 2 (DRM2) to catalyze cytosine methylation (Böhmdorfer et al., 2014; Liu et al., 2018; Wierzbicki et al., 2009). RdDM primarily methylates small euchromatic TEs and DNA present at the edges of larger TEs, particularly those closer to 
genes (Zemach et al., 2013). Despite its function in euchromatin, RdDM rarely functions at protein-coding genes (Matzke and Mosher, 2014).

Genomic regions are targeted for 24-nt siRNA production by members of the CLASSY (CLSY) family of putative chromatin-remodeling factors (Zhou et al., 2018). The four CLSY family members direct Pol IV to chromatin in a series of partially-redundant relationships. CLSY1 and CLSY2 function with SAWADEE HOMEODOMAIN HOMOLOGOUE1 (SHH1) and are responsible for nearly all 24-nt siRNA production in leaves (Law et al., 2013; Zhou et al., 2021). In parallel, CLSY3 and CLSY4 are responsible for Pol IV activity at a subset of loci in flowers, including abundantly expressed loci in ovules (Zhou et al., 2021).

In most cases, 24-nt siRNAs target DNA methylation in cis, utilizing their perfect complementarity to maintain asymmetric $\mathrm{CHH}$ methylation after DNA replication (where $\mathrm{H}=\mathrm{A}$, $\mathrm{T}$, or C). However, exogenous 24-nt siRNAs are capable of triggering DNA methylation and transcriptional silencing with up to 2 mismatches between the siRNA and target locus (Fei et al., 2021), indicating that Pol IV-derived siRNAs might also function in trans. Widespread "surveillance" transcription by Pol V (Tsuzuki et al., 2020) suggests that much of the genome may be poised for trans-acting siRNAs and it was recently demonstrated that 24-nt siRNAs trans-methylate genes during pollen development (Long et al., 2021). However, the extent of trans-acting RdDM is not understood.

Mutation of the RdDM pathway has only subtle defects in Arabidopsis, while loss of RdDM in other species have dramatic impacts on reproduction (Chow et al., 2020). In B. rapa, which diverged from Arabidopsis approximately 14.5 million years ago (mya), loss of RdDM in the maternal sporophyte results in a high rate of seed abortion (Grover et al., 2018). Seed development is dependent on RdDM activity in the maternal sporophyte, suggesting that 24-nt siRNA production in diploid maternal tissues is critical for sustained development of the endosperm and/or embryo (Grover et al., 2018).

We previously characterized a set of loci with extremely high expression of 24-nt siRNAs in ovules (termed siren loci). Although siren loci comprise only 1-2\% of all 24-nt dominant loci in Brassica rapa (B. rapa) ovules, they account for $90 \%$ of siRNA accumulation (Grover et al., 2020). In contrast, accumulation of siRNAs at these loci is negligible in leaves and anthers. Siren siRNA expression is dependent on Pol IV, RDR2, and CLSY3, and siren loci are heavily methylated in ovules and developing seed coats, demonstrating that siren siRNAs act in cis through canonical RdDM (Grover et al., 2020; Zhou et al., 2021). In endosperm, there is moderate accumulation of siren siRNAs, but with a striking maternal bias and a dependency on siren siRNA expression in maternal tissues (Grover et al., 2020), suggesting that these extremely abundant siRNAs might be transported into the endosperm from surrounding maternal tissues.

To better understand the origin and consequences of RdDM during $B$. rapa reproduction, we investigated siren loci and the resulting siren siRNAs. Here we show that $B$. rapa siren loci preferentially map to genic and intergenic regions and in most cases only overlap TEs at their edges. At least two TE families are associated with siren loci, suggesting that a specific region of these TEs may induce siren formation in the adjacent intergenic region. One-third of siren loci overlap genes or gene fragments, and in some cases, related B. rapa genes are trans-methylated 
specifically in tissues where siren siRNAs are expressed. Transcript accumulation for some of these trans-methylated target genes is impacted by RdDM, suggesting that siren siRNAs regulate expression of protein-coding genes during reproduction.

\section{RESULTS}

\section{Ovules and seed coats produce abundant non-TE 24-nt siRNAs}

To better understand the relationship between TEs and siRNA production in reproductive tissues, we manually annotated $415 \mathrm{TE}$ families in the $B$. rapa R-o-18 genome, including families that are lineage-specific and poorly-conserved. Approximately $40 \%$ of the $B$. rapa genome is composed of TE sequence compared to $21 \%$ for Arabidopsis (Wang et al., 2011). While $58 \%$ of leaf 24-nt siRNAs mapped to annotated TEs, only $13 \%$ of ovule 24-nt siRNAs mapped to TEs (Figure 1A). A similarly low percentage of seed coat 24-nt siRNAs mapped to TEs (12-13\%), whereas the percentage was higher in endosperm (43\%) and embryo (55\%). Consistent with the low rate of TE-derived sequences, the percentage of 24-nt siRNAs that map to only one genomic position was much higher in ovule and upper seed coat compared to leaf, endosperm, or embryo (Figure 1B).

We next asked whether uniquely-aligning 24-nt siRNAs from ovule differ in their chromosomal distribution from uniquely-aligning 24-nt siRNAs from leaf. For this analysis we used an earlier version of the $B$. rapa R-o-18 genome that had been assembled into pseudochromosomes. As a proxy for pericentromeric regions and large gene-poor heterochromatic regions we used Gypsy retrotransposon coverage and "graveyard regions", TE-rich regions in which synteny has been lost between Arabidopsis and B. rapa (Freeling et al., 2008). As expected, graveyard regions overlapped with high Gypsy element coverage. In each pseudochromosome, uniquely-mapping ovule 24-nt siRNAs occurred in discrete peaks that correspond to previously described siren loci (Grover et al., 2020) (Figure 1C, Supplemental Figure 1A). This pattern was highly reproducible (Supplemental Figure 1B) and persisted when reads were mapped to a TE-masked genome (Figure 1C, Supplemental Figure 1C). This pattern differs strikingly from uniquelymapping leaf 24-nt reads, which align evenly but at a much lower density across chromosomes (Figure 1C, Supplemental Figure 1A).

To determine whether aligning to non-TE genomic regions is a special property of siren loci, which produce abundant 24-nt siRNAs in ovules and seed coats, or is a general feature of 24-ntgenerating loci from ovule, we compared siRNA abundance and TE coverage in 24-nt windows tiled across all siRNA loci. In order not to bias the analysis against reads aligning to nearlyidentical repetitive elements, reads were aligned using ShortStack, which assigns multiplyaligning siRNAs to a position probabilistically, based on the local clustering of uniquelymapping reads (Johnson et al., 2016). In ovules, there is a striking negative correlation between read counts and average TE coverage (Figure 2A). In contrast, the average TE coverage in leaf is much higher, and only the few exceptional windows with high read count have low TE coverage (Figure 2B). To determine whether ovule 24-nt-loci overlap with genes, read counts were plotted against average gene coverage, and in this case ovule read count positively correlated with average gene coverage, with almost $30 \%$ of windows overlapping annotated genes (Figure 2A), whereas for leaf 24-nt loci only the few exceptional windows with high read 

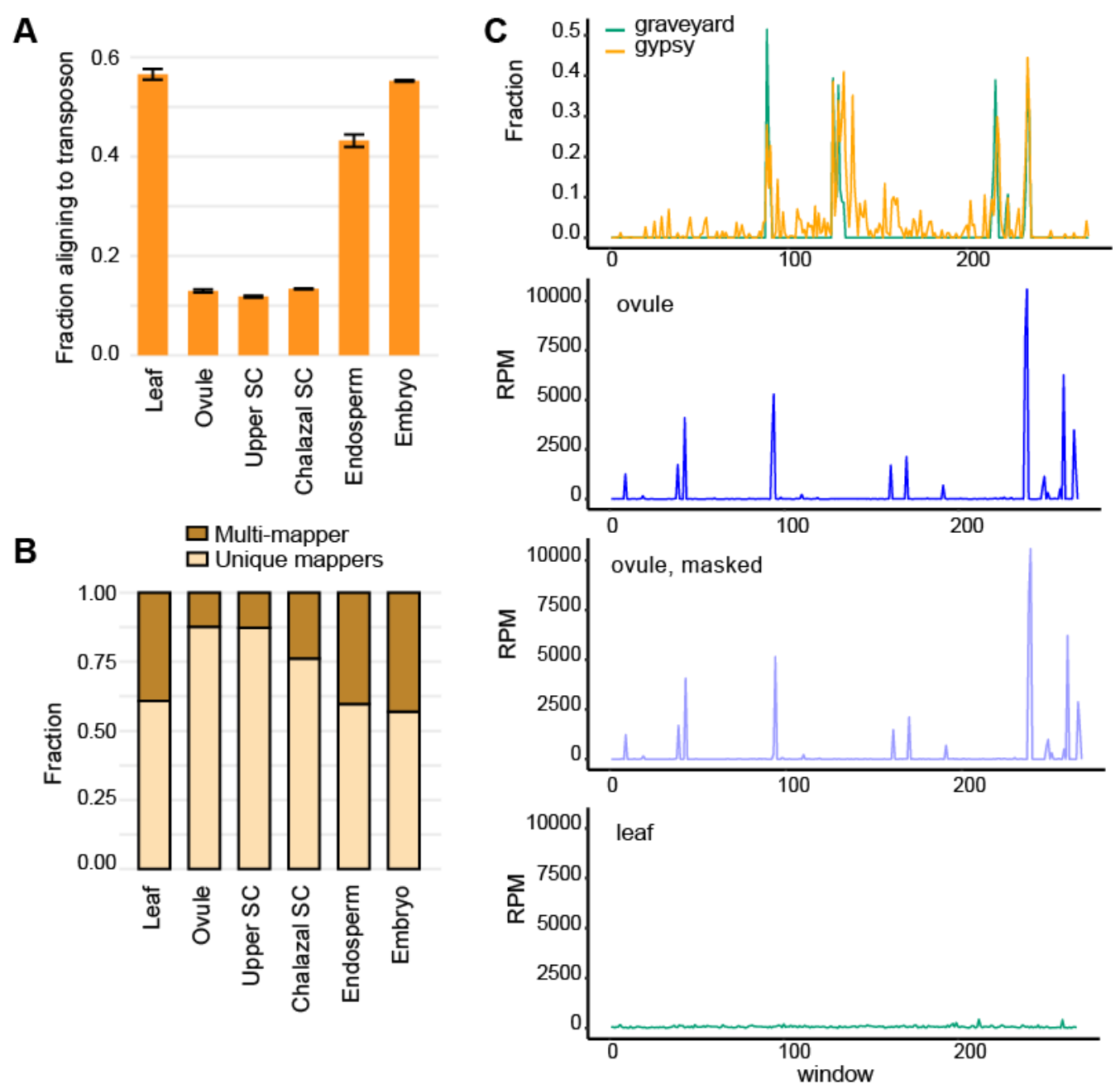

Figure 1. B. rapa ovule 24-nt siRNAs predominantly map to non-repetitive regions and siRNA hotspots.

(A) The fraction of 24-nt siRNAs from various tissues and organs aligning to annotated TEs. Error bars depict the standard error of the mean. SC, 17 days post fertilization seed coat. (B) Fraction of 24-nt siRNAs from different tissues and organs mapping uniquely or to multiple genomic locations. (C) Distribution of Gypsy elements, graveyard genes, and 24-nt siRNAs across $100 \mathrm{~kb}$ windows of pseudochromosome A01 of R-o-18, v1.2. Results for pseudochromosomes A02-A10 can be viewed in Supplemental Figure 1. Graveyard regions were identified as regions of at least 12 B. rapa genes without syntenic $A$. thaliana orthologs. Graveyard and Gypsy retrotransposon data are plotted as the fraction coverage for each window. Read counts for ovule and leaf siRNAs mapped to the full or a TE-masked genome.

count had a similarly high average gene coverage (Figure 2B). These results indicate that while siRNA-generating loci frequently overlap TEs, in ovules more siRNA production occurs from non-TE regions of these loci, suggesting that the RdDM pathway in ovule and seed coat may not primarily function to target TEs for silencing.

\section{Most siren loci are adjacent to TES}

Although most siRNA production in ovules arises from non-TE regions, our previous work demonstrated that siren loci are enriched for some classes of TEs (Grover et al., 2020). We 
therefore analyzed overlap between TEs and siren loci in greater detail. In this study, we defined $B$. rapa siren loci as the most abundant 157 loci with uniquely-aligning 24-nt reads from ovule sRNA data (Dataset 1). Each locus has $>5,000$ uniquely-aligning 24-nt reads (1,249-24,379 RPM). While $67 \%$ of siren loci overlap an annotated TE, this overlap encompasses only a small fraction of the siren length $(8.3 \%$, median) (Supplemental Table 1, Supplemental Figure 2A), with TE coverage being generally highest at the edge of the siren (Supplemental Figure 2B). In contrast, the peak in the number of uniquely-aligning 24nt reads occurs 200-300 bp into the siren (Supplemental Figure 2C), a point at which the average TE coverage has dropped by half. When we examined siren loci for overlap with specific families of TEs, two families were found to be significantly enriched - the non-autonomous Helitron family rnd-5_family-1287 (hereafter termed Persephone $)$ overlaps $19 \%$ of siren loci $(\mathrm{z}=$ $55 ; \mathrm{p}=0$; based on 10,000-randomly shuffled windows excluding genes) and the non-autonomous hAT Bra_hATl overlaps $4.5 \%$ of sirens $(\mathrm{z}=24 ; \mathrm{p}=0)$.

The reference sequence used to define the

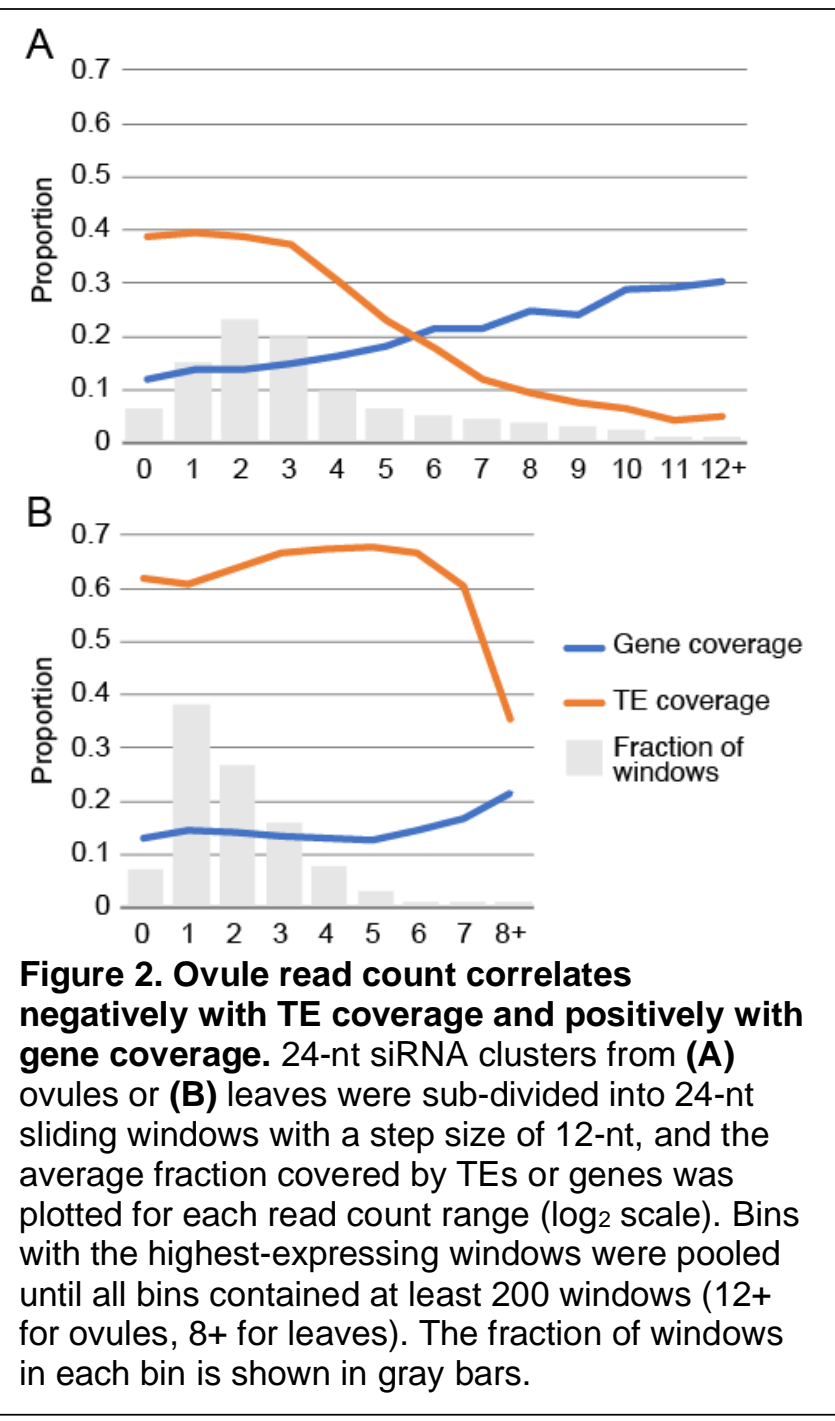
Persephone family ends with a short GC-rich hairpin followed 8 bp later by CTAG(T) (Dataset 2), as is characteristic of Helitrons (Yang and Bennetzen, 2009). It also contains internal repeated sequences, but does not carry exapted gene fragments as some Helitrons do. This family has complex structures as a consequence of insertions, truncations, and deletions between pairs of internal repetitive sequences. Despite this variation, alignment of the 27 Persephone elements associated with siren loci identified a 74-bp sequence retained in each instance (Figure 3A, Supplemental Figure 3), suggesting that this sequence is responsible for siren behavior in ovules. To determine if Persephone elements are generally associated with 24-nt siRNA clusters, eight additional Persephone family members were examined. Three of these were associated with 24-nt siRNA clusters that did not reach the expression threshold of a siren locus, while five had extremely low siRNA accumulation despite having complete or nearly complete copies of the element (Supplemental Figure 3). This observation suggests that the 74-nt sequence might be necessary, but not sufficient, for abundant siRNA production, and that genomic context may also be critical to triggering siRNA hot spots in ovule. 


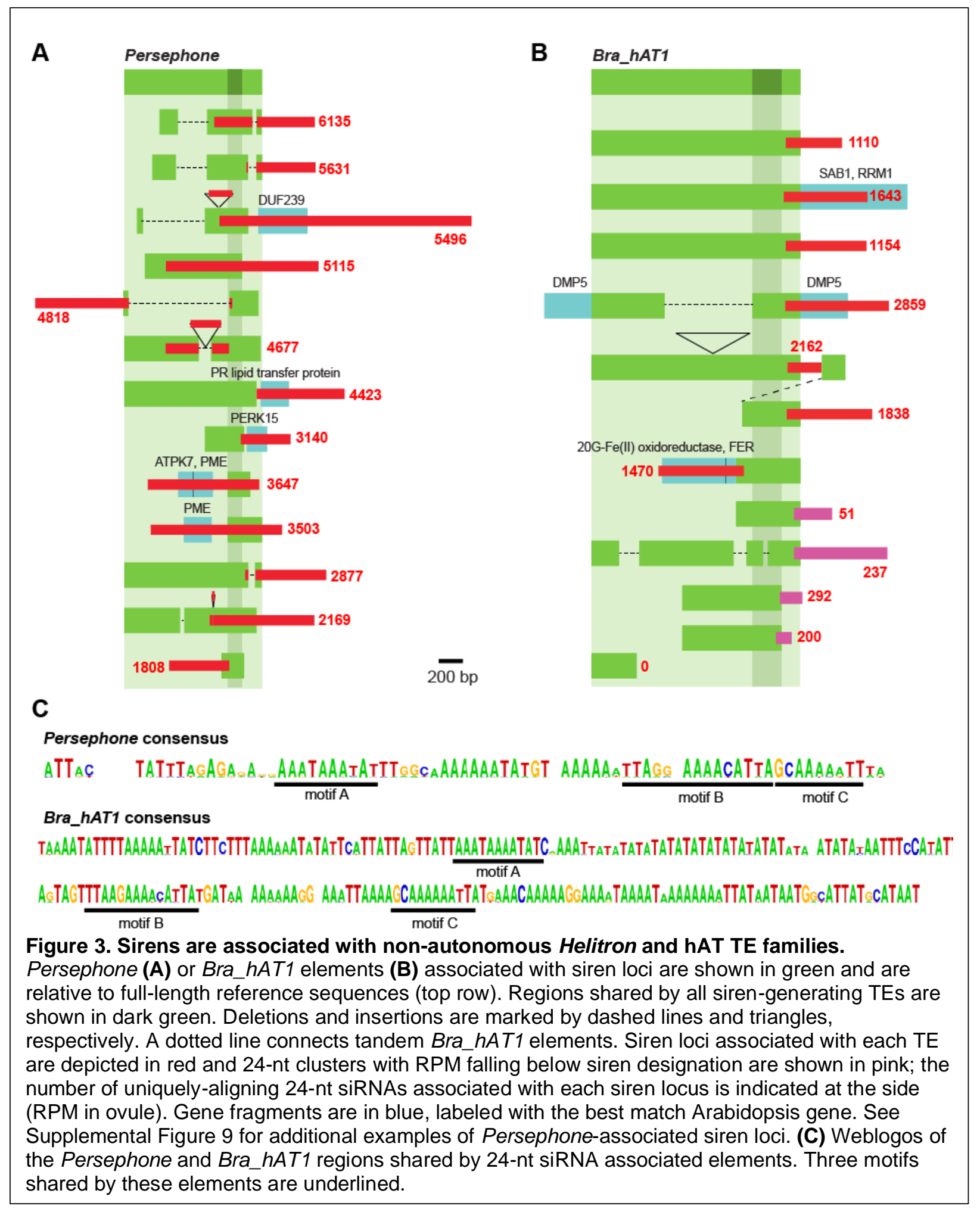

We performed similar assessment of the seven Bra_hATl-associated sirens relative to a reference sequence that retains the $8 \mathrm{bp}$ target-site duplication characteristic of this superfamily (Dataset 2). Three of the siren-associated Bra_hAT1 elements are full-length while the others have a variety of internal deletions, insertions, or truncations (Figure 3B). In general, siren loci overlap 50-150 nt with one end of the Bra_hATl element, and then extend hundreds of nucleotides in the 
same direction. Only 1 siren locus extends in the opposite direction, but this overlaps the same end of Bra_hAT1. To determine whether Bra_hATl is generally associated with 24-nt hot spots in ovule, we used blastn to find the five best additional matches to the Bra_hATl reference sequence. In 4 cases these $B r a \_h A T 1$ elements were associated with 24-nt clusters. In the final case only the end not associated with siren siRNA production is retained, and no 24-nt siRNAs are present (Figure 3B). Bra_hAT1 elements associated with sirens or 24-nt clusters all share an $\sim 200$ bp region in common, suggesting that a sequence within this region might be sufficient to trigger 24-nt siRNA expression in flanking sequences.

We looked for possible motifs shared by the 74-bp Persephone sequence and the 200-bp Bra_hATl sequence. Runs of adenine are present in both sequences, which is consistent with the presence of A/T rich sequences flanking Pol IV-transcribed regions (Li et al., 2015). Three specific sequence motifs are also present in the same order in each sequence (Figure 3C). Motifs $\mathrm{B}$ and $\mathrm{C}$ are adjacent in the 74-bp Persephone sequence and duplicated in one member of this family. The average percentage identity for these 3 motifs is higher in the 30 Persephone elements associated with sirens and 24-nt clusters (95\% for both groups) than for the 5 Persephone elements without an associated 24-nt cluster (84\%), suggesting that these motifs might function in siren siRNA production.

\section{Ovule siren siRNAs are enriched for protein-coding sequences}

$30 \%$ of the highest-expressed 24-nt windows in B. rapa ovule overlap annotated genes, prompting us to investigate the relationship between ovule siren loci and annotated sequences in Arabidopsis. Previously we had characterized the 65 highest expressing Arabidopsis ovule siren loci (17, Dataset 3). Twenty-nine of these (43\%) overlap at least one annotated non-TE gene, including long non-coding RNAs and annotated pseudogenes (Supplemental Table 2). Because pseudogenes are often unannotated, we also overlapped siren loci with a list of 4771 pseudogenes predicted by Zou et al. (Zou et al., 2009). Twenty siren loci overlapped with pseudogenes from this list (31\%), a highly significant number since on average only 3.7 shuffled regions overlap pseudogenes (10,000 randomly shuffled windows; $z=8.9 ; p=0)$.

We next determined if $B$. rapa siren loci are also associated with pseudogenes or gene fragments. Fifty-seven of the 157 siren loci overlap features annotated as genes. The structure of the sirenoverlapped genes was compared with the most closely-related genes in Arabidopsis and B. rapa to determine whether the genes were complete. Twenty-five of the siren-overlapped genes were found to be substantially shorter and missing exons, suggesting they are gene fragments (Supplemental Table 1). To determine if other siren loci overlap unannotated gene fragments, we used the siren sequence as query in blastn and tblastx searches. Fifty-two of the 157 siren loci returned Arabidopsis or B. rapa genes at an e-value less than $10^{-08}$ (Supplemental Table 1), and generally, the peak in siren read count corresponds to the embedded gene fragment (Supplemental Figure 4). These results demonstrate that loci producing the vast majority of ovule siRNAs, siren loci, are enriched for genes, pseudogenes, or gene fragments in B. rapa and Arabidopsis. Endosperm siren siRNAs in rice also predominantly map to genic and intergenic regions rather than to TEs (Rodrigues et al., 2013). 


\section{Ovule siren siRNAs act in trans at homologous genes}

Two B. rapa siren loci contain gene fragments related to AT4G03930 (encoding PME42), a pectin methylesterase gene expressed during early silique development (Louvet et al., 2006) (Figure 4A, Supplemental Figure 4). Both siren loci also contain truncated Persephone elements, and one also carries a fragment related to AT3G27580, encoding a protein kinase that phosphorylates an auxin efflux carrier. In both siren loci the majority of 24-nt siRNAs overlap with the gene fragments and not the TE (Figure 4B, Supplemental Figure 4). These gene fragments have elevated $\mathrm{CHH}$ and $\mathrm{CHG}$ methylation in ovules relative to leaves, and this methylation requires siRNA production by RDR2 (Figure 4C-D). In contrast, methylation in the $\mathrm{CG}$ context is high in both ovule and leaves, and is not affected in $r d r 2-2$ (Figure 4C-D). Inspection of DNA methylation over the most closely related full-length PME gene in $\mathrm{B}$. rapa (A02g503100_BraROA) revealed high cytosine methylation in all sequence contexts specifically in the region with homology to the siren gene fragments (Figure 4E-H). Only methylation in the CG context is present in leaves and $r d r 2-2$ mutants, and it is present at a reduced level (Figure 4G), indicating that methylation at this gene is dependent on 24-nt siRNAs expressed in the ovule. To determine whether siRNAs produced from the two siren loci target methylation at the full-length gene, siren 19-26mer siRNAs were re-aligned to A02g503100_BraROA allowing up to two mismatches. $7.96 \%$ of reads from one siren locus (608 RPM) and $3.53 \%$ of reads from the other siren locus (270 RPM) realigned to A02g503100_BraROA, none of them perfectly (Figure 4F). In contrast, only 32 siRNAs (5 RPM) originate from A02g503100_BraROA, and almost half of these are 22-nt siRNAs. Taken together, these observations suggest that siRNAs produced by the siren-associated gene fragments target methylation at the PME gene in trans.

To identify putative $B$. rapa trans-regulatory targets genome-wide, we aligned siren-derived siRNAs to annotated protein-coding genes, allowing up to 2 mismatches between the siRNA and target locus. This list was filtered to remove siren loci that overlap annotated genes and transposable elements mis-annotated as protein-coding genes, resulting in 276 regions mapping to 265 genes. About half of the regions $(136,49 \%)$ showed evidence of ovule-specific RdDM based on increased $\mathrm{CHH}$ and $\mathrm{CHG}$ methylation at the region of siRNA realignment and lack of this methylation in leaves or $r d r 2$ ovules (Supplemental Figure 5A). The number of realigning 24-nt siRNAs is weakly correlated with the methylation level (Supplemental Figure 5B) despite the fact that most re-aligning siRNAs did not perfectly match the gene (Supplemental Figure 5C).

To investigate this pattern further, we curated a list of putative trans-methylation target genes based on the number of realigning reads, the extent of homology between the siren locus and the target locus, and evidence of RdDM (Supplemental Table 3). To limit redundancy between homeologs and members of large gene families, no more than two genes per siren locus were included. Methylation in all three contexts is higher at the region with remapping siRNAs than at the 100 bp flanking the region or at the rest of the gene (Figure 5A). CHH and CHG methylation are ovule-specific and require $R D R 2$ (Figure 5B), while CG methylation is moderately higher in wild-type ovules compared to leaves or $r d r 2$ ovules. The amount of $\mathrm{CHH}$ and $\mathrm{CHG}$ methylation is highly correlated at the region with re-aligned siren siRNAs (Figure 5C). Together, these results indicate that siren siRNAs function in trans to direct de novo methylation of proteincoding genes. 
bioRxiv preprint doi: https://doi.org/10.1101/2021.06.10.447945; this version posted June 10,2021. The copyright holder for this preprint (which was not certified by peer review) is the author/funder, who has granted bioRxiv a license to display the preprint in perpetuity. It is made available under aCC-BY-NC-ND 4.0 International license.

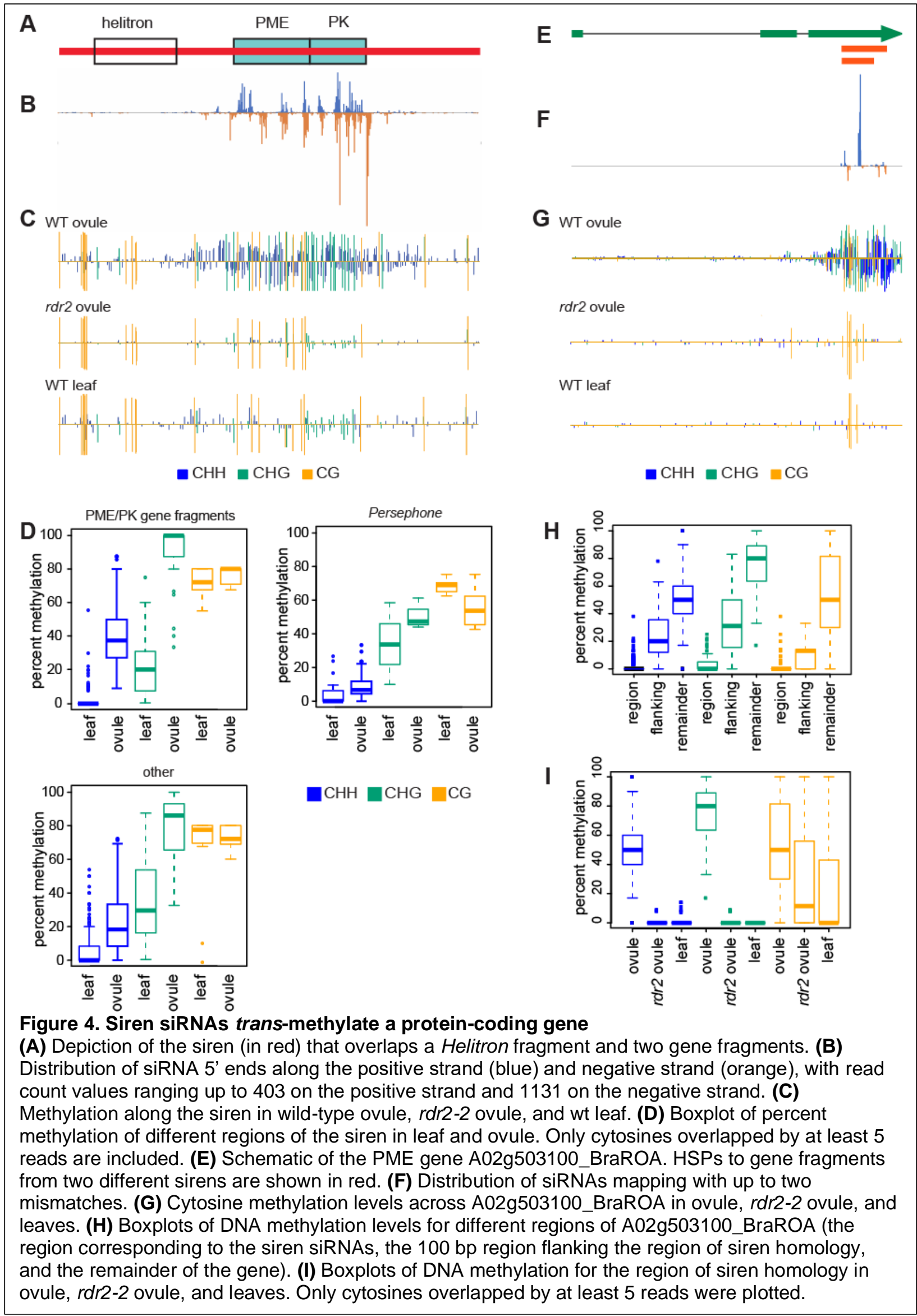

Burgess D, et al. 


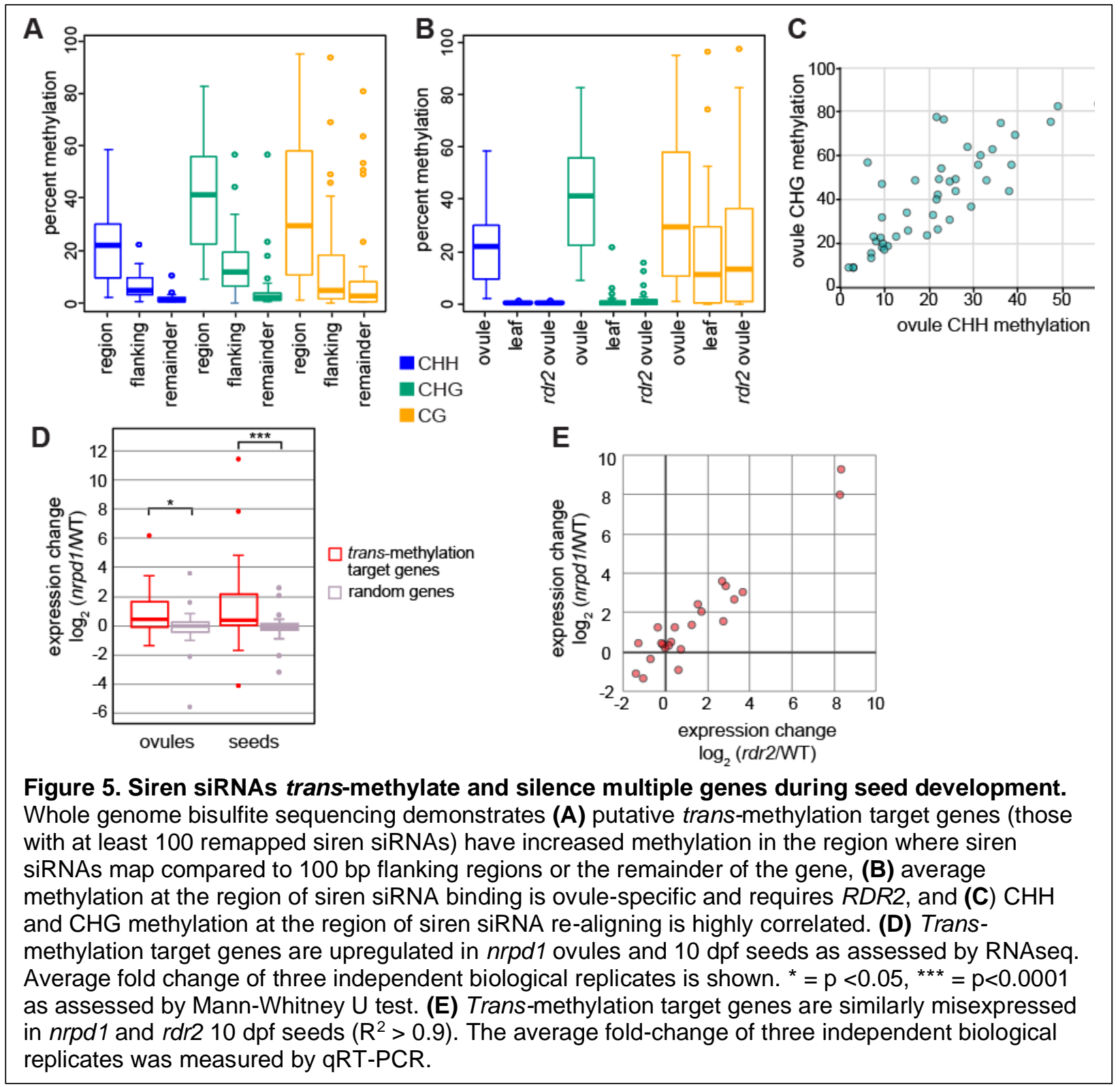

\section{Trans-acting siRNAs influence expression of genes}

Many gene bodies contain CG methylation, although the relationship between this methylation and transcriptional regulation is unclear (Bewick et al., 2016). RdDM-mediated non-CG methylation of promoter regions is most commonly associated with transcriptional silencing, although transcriptional activation can also occur (Matzke and Mosher, 2014; Williams et al., 2015). To understand whether siren siRNA trans-methylation of coding regions might influence expression of the targeted genes, we searched RNAseq data to compare transcript levels of wild type and nrpdl ovules before fertilization and seeds 10 days post fertilization (dpf). The transmethylation target genes are significantly upregulated in nrpdla ovules or young seeds relative to a set of randomly selected genes (Figure 5D, Supplemental Table 3). Transcript accumulation of A02g503100_BraROA, the PME gene described above, increases 74-fold in ovule and 2723-fold in young seeds. Using qRT-PCR on independent samples, we confirmed 
that many trans-methylation target genes are upregulated in nrpdl (Supplemental Table 4). We also observed upregulation in $r d r 2$, and this correlated with misexpression in $n r p d l$ (Figure 5E, Supplemental Table 4). While we cannot eliminate the possibility of an upstream regulator that is RdDM-sensitive, expression changes at many of the trans-methylation target genes suggests that siRNAs produced by siren loci silence expression of genes targeted in trans.

Fragments from specific gene families are shared by siren loci in B. rapa and Arabidopsis

B. rapa and Arabidopsis diverged approximately 14.5 million years ago (mya). This was followed by a whole genome triplication of the Brassica genus lineage 10.3 mya and subsequent extensive loss of duplicate genes (Cheng et al., 2013). We previously reported that there is limited synteny between Arabidopsis and B. rapa siren loci and no shared sequence between syntenous sirens (Grover et al., 2020), demonstrating the rapid evolution of siren loci. However, we have now found that while syntenic sirens share no sequence in common, they can be related to each other through the retention of non-overlapping gene fragments from an ancestral gene. For a quartet consisting of an Arabidopsis siren locus that is syntenically conserved at all three homeologous positions in $B$. rapa (Grover et al., 2020), the three B. rapa sirens contain exons of a DUF239 (neprosin peptidase domain) gene, while the syntenic Arabidopsis siren contains a different exon corresponding to the same gene (Figure 6). In Arabidopsis lyrata, a full-length DUF239-family gene exists in this position, suggesting that this gene was independently converted into a siren locus in B. rapa and Arabidopsis. Since the ancestral gene is no longer present in the $B$. rapa genome, and these siRNAs do not align with other DUF239 B. rapa genes, it may be that these siren loci have been syntenically conserved to maintain epigenetic structure at this position rather

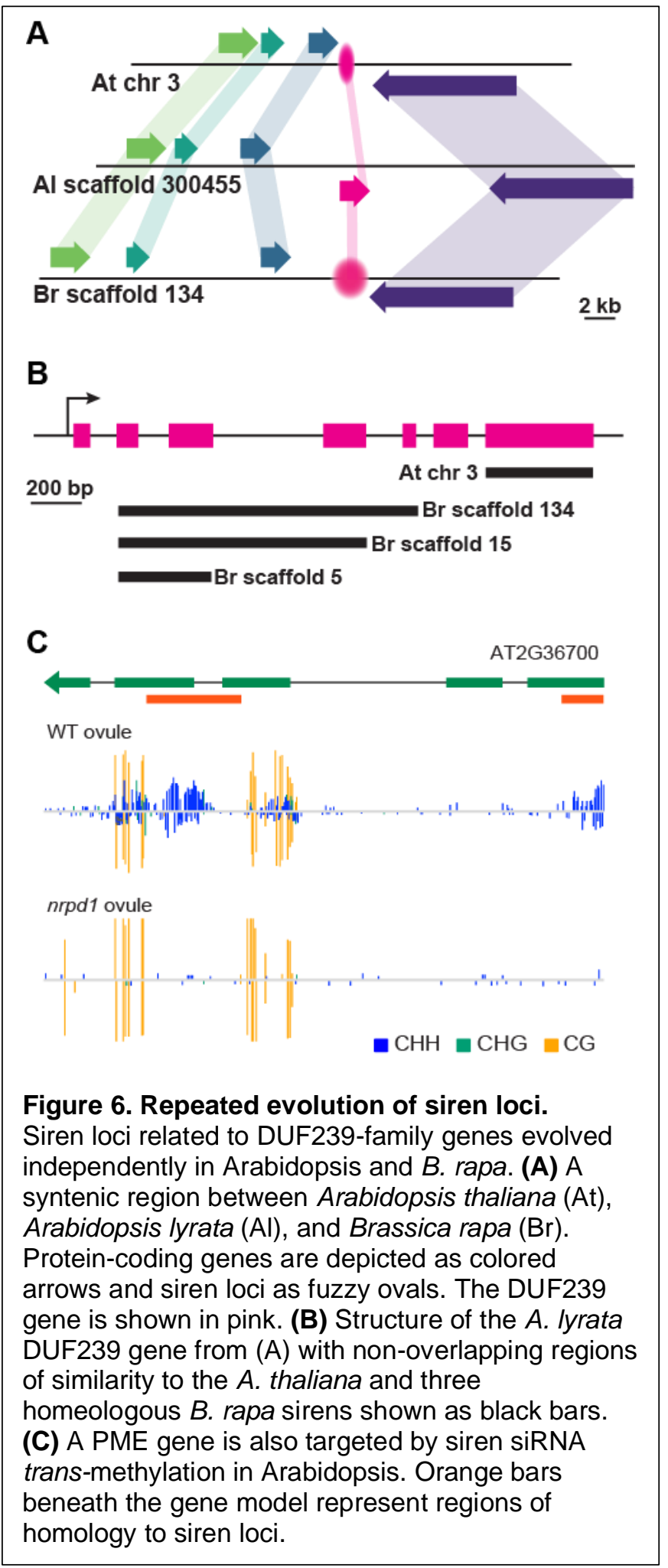


than for trans-methylation. However, there is no change in expression of proximal genes when siren siRNA production is eliminated, and therefore it remains unknown why siren character would have convergently evolved at this position.

Multiple non-syntentic siren loci in Arabidopsis and B. rapa also contain fragments of genes from the same gene families, and frequently from the same members of these gene families (Table 1, Supplemental Tables 1-2). For example, sixteen B. rapa and six Arabidopsis siren loci carry fragments from DUF239 domain genes, including three Arabidopsis siren loci and seven B. rapa siren loci that are most similar to the same DUF239 gene, AT5G18460. In addition to the two siren loci described above that trans-methylate PME gene A02g503100_BraROA, six additional B. rapa siren loci and three Arabidopsis siren loci overlap PME gene fragments. Although the PME family contains 66 members in Arabidopsis, gene fragments found in Arabidopsis and B. rapa siren loci are closely related to only 6 members of this family, which themselves fall into two groups of related sequences (Louvet et al., 2006).

To determine whether trans-methylation by siren siRNAs is conserved, we assessed ovule methylation at the three Arabidopsis PME genes with similarity to siren gene fragments. Two of these (AT1G11590 and AT2G36710) have methylation in all cytosine contexts across the entire gene and no change in methylation in $n r p d l$ ovules. In contrast, AT2G36700 shows strong NRPD1-dependent $\mathrm{CHH}$ and $\mathrm{CHG}$ methylation specifically in the region homologous to siren loci (Supplemental Figure 6C). RNAseq data demonstrates that AT2G36700 is upregulated 77fold in $n r p d 1$ ovules, 4.6 fold in clsy3 ovules, but is unchanged in $n r p d 1$ or $c l s y 3$ leaves (Zhou et al., 2021), suggesting that siren-mediated trans-methylation represses transcription of PME genes in both Arabidopsis and B. rapa. Multiple non-syntenic siren loci targeting the same genes or gene families in Arabidopsis and B. rapa suggests convergent evolution of siren siRNAmediated trans-methylation. Alternatively, conserved siren loci might be rapidly repositioned in the genome.

\section{Tissue-specific expression of siren siRNAs requires CLSY3 function}

To investigate factors responsible for expression of siren loci, we overlapped Arabidopsis ovule siren loci with 24-nt siRNA clusters from unopened flower buds of various siRNA mutants (Zhou et al., 2018). As expected, all 65 Arabidopsis siren loci were dependent on nrpdl. Additionally, 58 of these loci were dependent on $C L S Y 3$ and the remaining 7 loci were affected by the clsy3 clsy4 double mutant. Loss of CLSY1, CLSY2, or SHH1 had no impact on siren

\begin{tabular}{|c|c|c|c|c|}
\hline \multirow[b]{2}{*}{ Gene family/domain } & \multicolumn{2}{|c|}{$\begin{array}{l}\text { Number of siren loci with } \\
\text { homologous fragments }\end{array}$} & \multicolumn{2}{|c|}{$\begin{array}{c}\text { Number of genes most-similar to } \\
\text { siren gene fragments }\end{array}$} \\
\hline & B. rapa & Arabidopsis & B. rapa ${ }^{\mathrm{a}}$ & Arabidopsis \\
\hline TMK2 receptor-like kinase & 9 & 2 & $2(1)$ & 1 \\
\hline Pectin methylesterase & 8 & 3 & $7(6)$ & 3 \\
\hline DUF239-containing & 16 & 6 & $6(3)$ & 2 \\
\hline
\end{tabular}



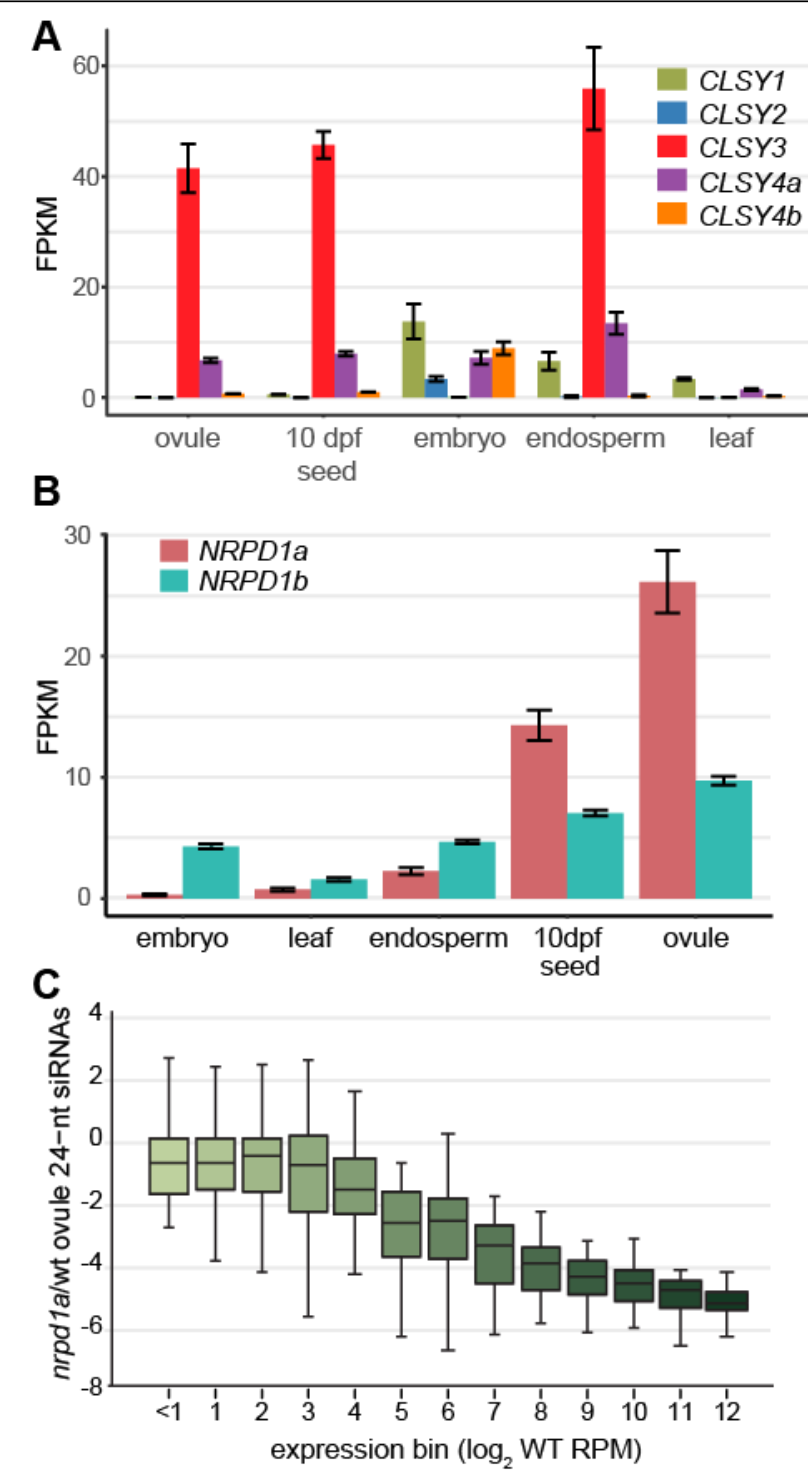

D

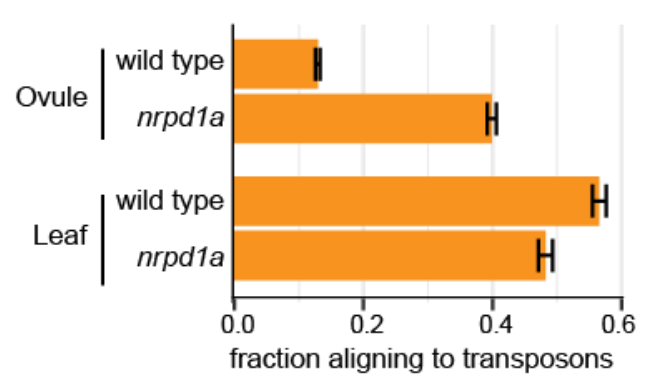

siRNA accumulation. Zhou et al have also established that siRNA production in ovules is dependent on CLSY3 and CLSY4, which are highly expressed in ovules (Zhou et al., 2021).

We therefore examined transcript accumulation of the CLASSY gene family in B. rapa. Following the Brassica whole genome triplication, one gene has been retained in $B$. rapa for CLSY1 (A08p019180.1_BraROA), CLSY2 (A10p025980.1_BraROA), and CLSY3 (A10p004670.1_BraROA), and two genes for CLSY4 (A07p011690.1_BraROA, A03p046540.1_BraROA). As in Arabidopsis, CLSY3 is the predominant family member expressed in ovule, with both $C L S Y 1$ and $C L S Y 2$ expressed at very low levels (Figure 7A). CLSY3 is also the predominant family member expressed in $10 \mathrm{dpf}$ seed and endosperm. Expression of CLSY3 is much higher than expression of CLSY4 in both ovule and endosperm, which is consistent with the stronger requirement for $C L S Y 3$ for siRNA production at siren loci in Arabidopsis (Zhou et al., 2021). In tissues lacking substantial siren siRNA accumulation, such as leaf and embryo, CLSY3 expression is low and CLSY1 is the predominant family member expressed.

In Arabidopsis, an 18-bp sequence is enriched at sites of CLSY3 binding (Zhou et al., 2021). We compared this sequence with the conserved motifs from Persephone and Bra_hATI elements. The reverse complement of the CLSY-binding sequence is highly similar to motif $\mathrm{B}$

Figure 7. CLSY3, CLSY4, and NRPD1a are associated with ovule-specific expression in $B$. rapa. Transcript accumulation of CLSY genes (A) and NRPD1 orthologs (B) in B. rapa. Error bars show the standard error of the mean from three RNAseq replicates. (C) Ovule loci producing abundant 24-nt siRNAs are more strongly impacted by the nrpd1a mutation. Outliers are not shown. (D) The fraction of 24-nt siRNAs aligning to TEs increases in the ovule nrpd1a mutant, but is unchanged in leaves. Error bars depict the standard error of the mean of three sRNA-seq replicates. 
shown in Figure 3C, particularly in the Persephone sequence, where it is immediately adjacent to motif C (Supplemental Figure 6). It is therefore likely that Persephone and Bra_hATl elements carry sequences associated with CLSY3 binding, allowing them to induce siRNA production specifically and abundantly in ovules.

\section{A paralog of NRPD1 has specialized for siren siRNA production in B. rapa}

We previously showed that 24-nt siRNAs from ovule are almost completely lost from a $B$. rapa $r d r 2$ mutant (Grover et al., 2018). However, some siRNAs remain in the B. rapa nrpd1-2 mutant, which has a missense mutation in the highly conserved Metal A binding site (Supplemental Figure 7A-B) (Haag et al., 2009; Huang et al., 2013). While earlier B. rapa genome assemblies included only one NRPD1 gene, an improved genome assembly includes a second NRPD1 gene, providing a potential source for Pol IV activity in the nrpd1-2 mutant. The two NRPDI paralogs are differentially expressed (Figure 7B), with ovule expressing relatively more of the copy corresponding to nrpd1-2 (BraA.NRPD1a, A09p015000.1_BraROA), while leaves and the filial tissues (embryo and endosperm) predominantly express the second copy (BraA.NRPDIb, A08g503970.1_BraROA). We therefore investigated whether NRPDIa might be specifically responsible for production of the abundant non-TE siRNAs in ovules. Figure 7C shows that there is a positive correlation between expression level of an siRNA locus in wild type and loss of siRNA accumulation in nrpd1-2. In contrast, no such correlation exists for $r d r 2$ or $n r p e 1$ (Supplemental Figure 7C). This correlation suggests that NRPDIa is required for expression of the most abundant siRNA loci in ovules. We also compared the fraction of siRNAs arising from TE or non-TE sequences and discovered that $n p r d 1-2$ ovules contain a TE-rich population of siRNAs that is similar to the siRNA population in leaves (Figure 7D), indicating that these TEenriched loci are not influenced by NRPDIa. Together these observations suggest that NRPDIa specifically impacts expression of siren siRNAs in $B$. rapa ovules, while $N R P D 1 b$ is associated with expression of canonical RdDM loci.

\section{DISCUSSION}

The canonical RdDM pathway is well-described in plants as a pathway for control of TEs (Matzke and Mosher, 2014; Stroud et al., 2013). Here we show that in ovules, the abundant population of 24-nt siRNAs generated from siren loci is more frequently associated with pseudogenes and gene fragments. Production of siRNAs from siren loci is associated with expression of the putative chromatin remodeler CLSY3 and one of two copies of NRPD1. We conclude that these siRNAs induce $\mathrm{CHH}$ and $\mathrm{CHG}$ methylation in trans at closely-related genes from the following observations: 1) the methylated region(s) closely correspond to the gene fragment embedded in the siren(s);2) methylation is present in the ovule, where siren siRNAs are produced, but not in leaves; 3) both siren siRNA production and transmethylation are RDR2dependent, despite the fact that few siRNAs originate from the trans-methylated region itself; and 4) whether a closely-related gene is trans-methylated correlates well with the number of siRNAs originating from the siren that are able to realign with no more than two mismatches. The recent observation of widespread Pol V transcription (Tsuzuki et al., 2020) provides a plausible mechanism for siRNA-mediated recruitment of methyltransferases to target genes in trans, although Pol II might also create a scaffold for these siRNAs to function (Zheng et al., 2009). 
Some siren loci appear to derive from the pseudogenization of a once functional gene, based on the presence of a likely functional copy at a tandem position or at a syntenic position in close relatives. Although we observed a correlation between non-functional gene fragments and siren loci, we cannot determine which characteristic is causal. RdDM might enable initial pseudogenization or might be recruited by non-coding pseudogenic transcripts. Other siren gene fragments appear to be insertions relative to Arabidopsis, perhaps formed by the addition of filler DNA during double-strand break repair, as has been proposed for gene fragments captured by Helitrons (Kapitonov and Jurka, 2007). Double-strand break repair itself has been associated with the production of 21-nt siRNAs in the vicinity of the break (Wei et al., 2012), and these could in turn function to trigger chromatin modifications that initiate RdDM (Cuerda-Gil and Slotkin, 2016).

Siren-like loci that produce highly abundant 24-nt siRNAs were recently described in Arabidopsis tapetum, somatic cells surrounding the male germline (Long et al., 2021). It is not reported whether these loci carry gene fragments, however siRNAs produced from these sites function in trans to trigger methylation and silencing of protein coding transcripts. Like the ovule siren siRNAs described here, siRNAs from abundant tapetal loci also require CLSY3 (Zhou et al., 2021; Long et al., 2021), raising the possibility that similar siren-induced transmethylation functions in both maternal and paternal somatic tissue surrounding the germline. On the paternal side, siRNAs are produced in tapetum and trigger methylation in the male meiocyte. We have previously proposed that siren siRNAs move from maternal integuments to the developing endosperm, but do not influence methylation in the embryo (Grover et al., 2020; Chakraborty et al., 2021), however it remains to be tested where trans-methylation occurs. Importantly, the ability to function despite mismatches between the siRNA and the target locus indicates that maternally-derived siren siRNAs could function on both maternally- and paternally-derived alleles in endosperm. Imbalance between the dosage of maternal siRNAs and paternally-derived targets could contribute to maternal- or paternal-excess seed phenotypes (Lu et al., 2012). Trans-methylation at closely related sequences also implies that a single siren locus could impact diverging homeologous sequences following whole genome duplication.

Tapetal siren-like siRNAs silence transcription of a Gypsy retrotransposon through transmethylation of its LTR (Long et al., 2021). We also detected transcriptional changes for many of the trans-methylated genes in nrpdla ovules and young seeds (Figure 5DE). Genes targeted by siren siRNAs are more frequently upregulated in the mutant, suggesting that siren-induced transmethylation suppresses gene expression. Gene body methylation in the CG context occurs most often in moderately transcribed genes, but its effect, if any, on transcription is controversial (Bewick et al., 2016; Zilberman et al., 2007). Genes with heavy methylation tend to be more upregulated in a metl mutant, suggesting that methylation within gene bodies could impede transcript elongation (Zilberman et al., 2007). However, unlike the trans-methylated genes in this study, gene body methylation is absent from the first $2 \mathrm{~kb}$ and last $1 \mathrm{~kb}$ of genes (Zilberman et al., 2007). The trans-methylated genes in this study also differ in being highly methylated in $\mathrm{CHH}$ and $\mathrm{CHG}$ contexts, and while CG methylation of genes has been shown to correlate with expression, CHG methylation anticorrelates with expression (Schmitz et al., 2013). In Arabidopsis lyrata, genes gaining gene body CHG methylation in endosperm are associated with reduced gene expression, and the magnitude of increase in gene body $\mathrm{CHG}$ methylation in 
maternal alleles positively correlates with increased expression bias in favor of the paternal allele (Klosinska et al., 2016). Thus, siren-induced non-CG trans-methylation in gene bodies may be transcriptionally repressive.

We particularly noted that three gene families are associated with multiple sirens in both $B$. rapa and Arabidopsis: PMEs, TMK receptor-like kinases, and DUF239 genes. In each case the same phylogenetic group within these families is associated with siren loci, further suggesting functional significance of siren association. A reduction of only $18 \%$ in PME activity in a pollenspecific isoform is sufficient to affect pollen tubes, resulting in reduced fertilization (Jiang et al., 2005). Thus, siren-generated siRNAs could play a critical role in regulating the expression of PME genes, including the regulation of multiple closely-related family members within this large family. Siren loci may also play some additional unknown function as shown by the existence of a set of siren loci that are syntenically conserved in Arabidopsis and all three B. rapa homeologous positions that are related to each other through an ancestral DUF239 gene, but have no sequence homology to each other and no longer have strong sequence homology to any DUF239 genes existing in their own genome.

Whether their function is primarily trans-methylation or something else, hints of the importance of siren siRNAs come from the phenotype of the B. rapa nrpd1-2 mutant, which has a severe seed abortion phenotype (Grover et al., 2018). nrpd1-2 ovules lack siren siRNAs but produce most canonical 24-nt siRNAs (Supplemental Figure 6AB), indicating that seed production relies on siren siRNAs in B. rapa. Interestingly, the nrpdl-2 phenotype is determined by the maternal sporophytic genotype (Grover et al., 2018), supporting a model whereby maternallyproduced siRNAs cause trans-methylation in the endosperm to enable its proper development (Kirkbride et al., 2019).

Although a few siren loci are centered on a TE, most siren loci overlap a TE at the edge of the locus and not in the region where the majority of 24-nt siRNAs are produced (Supplemental Figure 2). Two specific families of TEs, the Helitron Persephone and the non-autonomous Bra_hATl element, are associated with almost a quarter of siren loci. For both TEs, additional family members were also associated with 24-nt siRNA clusters, suggesting that these elements may include motifs capable of inducing the production of siRNAs in adjacent sequences in the ovule. Alternatively, the siren environment might be favored for insertion by these elements. However, we believe this less likely because for both families, tandem copies of the TE resulted in the formation of independent sirens. In addition, for both TEs, internal deletions amongst family members reduced the relevant sequence to a very small region (74 bp for Persephone and 200 bp for Bra_hAT1). A Bra_hAT1 element retaining the wrong end of the element was the only family member not associated with 24-nt clusters. The region of these elements that is associated with siren siRNA production includes sequences similar to a CLSY3 binding motif (Supplemental Figure 6), suggesting a novel mechanism for transposons to produce epigenetic variability.

TEs have been exapted for a number of functions (reviewed in (Lisch, 2013)), including providing regulatory elements, placing genes under epigenetic control, and serving as the source of new protein-coding genes. Here we show that certain TEs may be able to induce large amounts of siRNAs to be made from adjacent sequences in an organ-specific fashion. When 
these adjacent sequences include gene fragments, siRNAs arising from the gene fragment can trans-methylate and transcriptionally silence closely-related genes. Such regulation could account for the sporophytic requirement of RdDM for seed development in B. rapa.

\section{MATERIALS AND METHODS}

\section{Biological material}

Brassica rapa subsp. trilocularis, R-o-18 genotype, was used for all experiments. Unfertilized ovules were collected less than $24 \mathrm{~h}$ prior to anthesis. B. rapa RdDM mutants were obtained from a TILLING population, then backcrossed six times to the parental line, as previously described (Grover et al., 2018). RdDM mutant lines braA.nrpd1.a-2, braA.rdr2.a-2, and braA.nrpe 1.a-1 are referred to as nrpdla, $r d r 2$, and nrpe1, respectively.

Earlier drafts of the B. rapa R-o-18 genome were used for some analyses, as noted in the text. All gene names correspond to the publicly-released v2.3 genome (NCBI GCA_017639395.1).

\section{Analysis of TEs}

B. rapa TEs were annotated as previously described (Grover et al., 2018), with the addition of RepBase (Bao et al., 2015) Brassica and Arabidopsis repetitive elements updated through vol 20, issue 3 and ten $B$. rapa TRIM elements (Gao et al., 2016). The TEs used in the annotation also included 1519 TEs from a RepeatModeler-generated dataset (Cheng et al., 2016); 1005 of these have been classified to family, 658 of which we have manually-annotated to generate full-length exemplars (Dataset 4). Detailed methods for transposon annotation are in the Supplemental Methods. Masked B. rapa and Arabidopsis genomes were generated by running RepeatMasker (v. 4-0.5) on this combined library of Brassica and Arabidopsis repetitive DNA elements, resulting in masking of $41.53 \%$ of the $B$. rapa $\mathrm{R}-0-18 \mathrm{v} 2$ genome and $20.25 \%$ of the Arabidopsis v10 genome.

Bra_hAT1 and Helitron rnd-5_family-1287 (Persephone) elements were aligned to the fulllength exemplar sequences (Dataset 2) using Muscle (https://www.ebi.ac.uk/Tools/msa/muscle/), with the alignment manually corrected based on blastn alignments between each individual family member and a full-length exemplar.

\section{Small RNA alignment}

B. rapa 19-26 bp small RNA reads were prepared, processed and filtered for structural and noncoding RNAs as previously described (Grover et al., 2020). Arabidopsis small RNA reads from publicly available databases were processed in parallel.

Small RNA reads were aligned to B. rapa genotype R-o-18 v2 using Bowtie (Langmead et al., 2009) under conditions permitting only perfectly-matched unique alignments (-v $0,-\mathrm{m} 1)$ or under conditions in which perfectly-matched reads multiply-aligning up to 49 times are positioned by ShortStack (Johnson et al., 2016) based on local densities of uniquely-aligning reads (--mismatches 0, --mmap u). RPM calculations were based on the number of alignable 19$26 \mathrm{nt}$ siRNAs in a library.

To re-align siren siRNAs, 19-26 nt Shortstack-aligned reads at siren loci were captured from the 
bamfile using the BEDTools intersect command, converted into a fastq file using the samtools fastq command, and realigned to CDS sequences using bowtie but allowing up to 2 mismatches (-v 2). The number of realigning reads and perfectly aligning reads was parsed from the data.

\section{Siren locus analysis}

24-nt siRNA ovule reads were size-selected and clustered using BEDTools v2.25.0 merge (Quinlan and Hall, 2010) and unix commands. Overlapping 24-nt siRNA clusters with at least 10 reads were merged when separated by no more than 100 bp. B. rapa clusters with at least 5000 reads (1245 RPM) were defined as siren loci, and an equivalent RPM was used to define the 65 Arabidopsis ovule siren loci (Datasets 1, 3). These siren loci are similar but not identical to previously reported ovule "core" siren loci (Grover et al., 2020). For a positional profile of 24-nt siRNA 5' ends mapping across sirens, a bedfile of 24-nt reads aligned to each siren using ShortStack was generated using the BEDTools intersect command; for each strand the number of 5 ' ends at each position was counted using a custom perl script.

Overlap between siren loci and genomic features (TEs, pseudogenes, CLSY-dependent loci) was determined using the BEDTools intersect command. To determine the statistical significance of overlaps with pseudogenes and TEs, 10,000 sets of non-overlapping genomic intervals of matching size were generated using BEDTools shuffle (-noOverlapping) and intersected with the pseudogene or TE feature. Genes were excluded from matching genomic intervals for the TE shuffle.

TE coverage across siren loci was averaged over $100 \mathrm{bp}$ windows starting at each end and preceding in 50 bp steps; at least five siren loci were included in each averaged window. TE coverage in each window was calculated using the BEDTools coverage command. The average fraction of siren 24-nt siRNAs in each $100 \mathrm{bp}$ window was determined using the BEDTools intersect command.

Syntenic orthologs between Arabidopsis and B. rapa were identified as previously described (Grover et al., 2020). The closest homologous Arabidopsis and B. rapa genes (e-value $<10^{-08}$ ) were identified with blastn and tblastx (performed using Blastall) with siren sequences or genes overlapped by siren sequences as queries. Genomic regions carrying siren loci were compared to genomic regions carrying best hit Arabidopsis and B. rapa genes with GEvo (Lyons et al., 2008) using genomes that had been annotated for siren loci and TEs.

\section{Chromosomal distribution analysis}

24-nt uniquely-aligning siRNA clusters with at least 10 overlapping reads were mapped to R-o18 v1.2 pseudochromosomes in 100,000 bp windows. A TE-masked genome was generated using RepeatMasker. Pericentromeric and large gene-poor heterochromatic regions were inferred using Gypsy retrotransposon coverage and graveyard regions, TE-rich regions in which synteny has been lost between Arabidopsis and B. rapa. Graveyard regions were identified using a perl script to identify $B$. rapa regions in which at least 12 contiguous genes lack an Arabidopsis ortholog. The BEDTools coverage command was used to determine the fraction of each window covered by graveyard regions or gypsy retrotransposons. Leaf read counts were normalized to ovule read counts based on the number of alignable 19-26 nt sRNAs in the libraries. 


\section{DNA methylation analysis}

B. rapa leaf and ovule bisulfite sequencing was performed and analyzed as previously described (1) using a WGBS Snakemake workflow (Grover, 2019). All bisulfite conversion rates were at least $99 \%$. Percentage methylation for each target gene was summarized over the following regions: the siren target region, a $100 \mathrm{bp}$ region flanking the siren target, and the remainder of the gene. The target gene region was defined using blastn between the siren and the target gene (word size 11; match/mismatch scores 2,-3; gap opening penalty 5; gap extension penalty 2).

Arabidopsis ovule methylation (NCBI GEO SRR13404120-SRR13404122, SRR13404131) was visualized with CoGe LoadExp+'s Methylation Analysis Pipeline using Bismark (Grover et al., 2017).

\section{mRNA-seq data analysis}

RNAseq from ovules and $10 \mathrm{dpf}$ seeds were obtained from SRA accession SRP132223 (Grover et al., 2018). Trimmed RNAseq reads were aligned to the R-o-18 v2 genome using STAR v2.5.4b (Dobin et al., 2013). Reads overlapping annotated genes were counted using htseq-count (Anders et al., 2015). Replicate consistency was checked by principle component analysis on rlog-transformed counts generated by DESeq2 (Love et al., 2014). Differentially expressed genes and FDR-corrected p-values were determined using DESeq2.

Differential expression of Arabidopsis PME gene AT2G36700 in clsy mutant ovules was retrieved from (Zhou et al., 2021).

\section{qRT-PCR}

B. rapa $10 \mathrm{dpf}$ seeds were collected for extraction of total nucleic acid (tNA) (White and Kaper, 1989). $2 \mu \mathrm{g}$ tNA were DNaseI digested with DNA-free kit DNase Treatment and Removal Reagents (Ambion) following the manufacturers" "rigorous" DNase treatment protocol. After removal of contaminating DNA, $20 \mu \mathrm{L}(1.8 \mu \mathrm{g})$ was incubated with $1 \mu \mathrm{L} 50 \mu \mathrm{M}$ random hexamers and $1 \mu \mathrm{L} 10 \mathrm{mM}$ dNTP mix at $65^{\circ} \mathrm{C}$ for 5 mins, followed by cDNA synthesis with SuperScript IV Reverse transcriptase (Invitrogen) according to the manufacturers' protocol with the following reaction: $5 \mu \mathrm{L}$ 5x SSIV buffer, $1 \mu \mathrm{L} 100 \mathrm{mM}$ DTT, $1 \mu \mathrm{L}$ RNaseOUT RNase Inhibitor $(40 \mathrm{U} / \mu \mathrm{L})$, and $1 \mu \mathrm{L}$ SuperScript IV Reverse transcriptase.

To quantify gene expression, qRT-PCR was carried out with $1 \mu \mathrm{L}$ of a 1:1 diluted cDNA, 0.625 $\mu \mathrm{L}$ of each $10 \mathrm{mM}$ gene specific primer (Supplementary Table 5), $12.5 \mu \mathrm{L} 2 \mathrm{x}$ SensiMix SYBR $\&$ Fluorescein master mix (Bioline) and $10.25 \mu \mathrm{L}$ nuclease-free water. Twenty-four putative trans-methylation target genes were selected for analysis; 23 were expressed at a detectable level. The melting curve from each primer set was checked to ensure the specificity of the qRTPCR reaction and the expression level of each transcript was normalized to ACTIN2. Three independent biological replicates were compared to determine fold-change and p-values.

\section{Accession numbers}

Sequence data from this article can be found in NCBI GEO under accession numbers SRR5886891-SRR5886893 (B. rapa ovule siRNAs), SRR10415409-SRR10415420 (B. rapa ovule WGBS), SRR6675211-SRR6675222 (B. rapa ovule and 10 dpf RNAseq), SRR5646727SRR5646729 (Arabidopsis flower bud siRNAs), and SRR13404120-SRR13404122, 
SRR13404131 (Arabidopsis ovule WGBS).

\section{ACKNOWLEDGEMENTS}

We are grateful to Dr. Eric Lyons and the CoGe team for development and maintenance of CoGe. We thank Dr. Damon Lisch for critical reading of an earlier draft and for suggesting that we look for transmethylation. The authors are grateful for support from the National Science Foundation (IOS-1546825 to RAM and MF) and the USDA National Institute of Food and Agriculture (AFRI 2021-67013-33797 to RAM and AFRI 2014-67013-21661, subaward C0471A-B to MF).

\section{AUTHOR CONTRIBUTIONS}

$\mathrm{DB}, \mathrm{MF}$, and RAM designed the research; all authors performed research; DB, HTC, MF, and RAM analyzed data; DB, MF, and RAM wrote the paper. All authors read and approved the manuscript.

\section{REFERENCES}

Anders, S., Pyl, P.T., and Huber, W. (2015). HTSeq--a Python framework to work with highthroughput sequencing data. Bioinformatics 31: 166-169.

Bao, W., Kojima, K.K., and Kohany, O. (2015). Repbase Update, a database of repetitive elements in eukaryotic genomes. Mob. DNA 6: 11.

Bewick, A.J. et al. (2016). On the origin and evolutionary consequences of gene body DNA methylation. Proc. Natl. Acad. Sci. U. S. A. 113: 9111-9116.

Blevins, T., Podicheti, R., Mishra, V., Marasco, M., Wang, J., Rusch, D., Tang, H., and Pikaard, C.S. (2015). Identification of Pol IV and RDR2-dependent precursors of $24 \mathrm{nt}$ siRNAs guiding de novo DNA methylation in Arabidopsis. Elife 4: e09591.

Böhmdorfer, G., Rowley, M.J., Kuciński, J., Zhu, Y., Amies, I., and Wierzbicki, A.T. (2014). RNA-directed DNA methylation requires stepwise binding of silencing factors to long non-coding RNA. Plant J. 79: 181-191.

Chakraborty, T., Kendall, T., Grover, J.W., and Mosher, R.A. (2021). Embryo CHH hypermethylation is mediated by RdDM and is autonomously directed in Brassica rapa. Genome Biol. 22: 140.

Cheng, F., Mandáková, T., Wu, J., Xie, Q., Lysak, M.A., and Wang, X. (2013). Deciphering the diploid ancestral genome of the Mesohexaploid Brassica rapa. Plant Cell 25: 15411554.

Cheng, F., Sun, C., Wu, J., Schnable, J., Woodhouse, M.R., Liang, J., Cai, C., Freeling, M., and Wang, X. (2016). Epigenetic regulation of subgenome dominance following whole genome triplication in Brassica rapa. New Phytol. 211: 288-299.

Chow, H.T., Chakraborty, T., and Mosher, R.A. (2020). RNA-directed DNA Methylation and sexual reproduction: expanding beyond the seed. Curr. Opin. Plant Biol. 54: 11-17. 
Cuerda-Gil, D. and Slotkin, R.K. (2016). Non-canonical RNA-directed DNA methylation. Nat. Plants 2.

Dobin, A., Davis, C.A., Schlesinger, F., Drenkow, J., Zaleski, C., Jha, S., Batut, P., Chaisson, M., and Gingeras, T.R. (2013). STAR: ultrafast universal RNA-seq aligner. Bioinformatics 29: 15-21.

Fei, Y., Nyikó, T., and Molnar, A. (2021). Non-perfectly matching small RNAs can induce stable and heritable epigenetic modifications and can be used as molecular markers to trace the origin and fate of silencing RNAs. Nucleic Acids Res. 49: 1900-1913.

Freeling, M., Lyons, E., Pedersen, B., Alam, M., Ming, R., and Lisch, D. (2008). Many or most genes in Arabidopsis transposed after the origin of the order Brassicales. Genome Res. 18: 1924-1937.

Gao, D., Li, Y., Kim, K.D., Abernathy, B., and Jackson, S.A. (2016). Landscape and evolutionary dynamics of terminal repeat retrotransposons in miniature in plant genomes. Genome Biol. 17: 7.

Grover, J. (2019). groverj3/wgbs_snakemake: Initial Release (Zenodo).

Grover, J.W., Bomhoff, M., Davey, S., Gregory, B.D., Mosher, R.A., and Lyons, E. (2017). CoGe LoadExp+: A web-based suite that integrates next-generation sequencing data analysis workflows and visualization. Plant Direct 1: 343.

Grover, J.W., Burgess, D., Kendall, T., Baten, A., Pokhrel, S., King, G.J., Meyers, B.C., Freeling, M., and Mosher, R.A. (2020). Abundant expression of maternal siRNAs is a conserved feature of seed development. Proc. Natl. Acad. Sci. U. S. A. 117: 1530515315.

Grover, J.W., Kendall, T., Baten, A., Burgess, D., Freeling, M., King, G.J., and Mosher, R.A. (2018). Maternal components of RNA-directed DNA methylation are required for seed development in Brassica rapa. Plant J. 94: 575-582.

Haag, J.R., Pontes, O., and Pikaard, C.S. (2009). Metal A and metal B sites of nuclear RNA polymerases Pol IV and Pol V are required for siRNA-dependent DNA methylation and gene silencing. PLoS One 4: e4110.

Havecker, E.R., Wallbridge, L.M., Hardcastle, T.J., Bush, M.S., Kelly, K.A., Dunn, R.M., Schwach, F., Doonan, J.H., and Baulcombe, D.C. (2010). The Arabidopsis RNAdirected DNA methylation argonautes functionally diverge based on their expression and interaction with target loci. Plant Cell 22: 321-334.

Huang, Y., Kendall, T., and Mosher, R. (2013). Pol IV-Dependent siRNA Production is Reduced in Brassica rapa. Biology 2: 1210-1223.

Jiang, L., Yang, S.-L., Xie, L.-F., Puah, C.S., Zhang, X.-Q., Yang, W.-C., Sundaresan, V., and Ye, D. (2005). VANGUARD1 encodes a pectin methylesterase that enhances pollen 
tube growth in the Arabidopsis style and transmitting tract. Plant Cell 17: 584-596.

Johnson, N.R., Yeoh, J.M., Coruh, C., and Axtell, M.J. (2016). Improved placement of multimapping small RNAs. G3 (Bethesda) 6: 2103-2111.

Kapitonov, V.V. and Jurka, J. (2007). Helitrons on a roll: eukaryotic rolling-circle transposons. Trends Genet. 23: 521-529.

Kirkbride, R.C., Lu, J., Zhang, C., Mosher, R.A., Baulcombe, D.C., and Chen, Z.J. (2019). Maternal small RNAs mediate spatial-temporal regulation of gene expression, imprinting, and seed development in Arabidopsis. Proc. Natl. Acad. Sci. U. S. A. 116: 2761-2766.

Klosinska, M., Picard, C.L., and Gehring, M. (2016). Conserved imprinting associated with unique epigenetic signatures in the Arabidopsis genus. Nat. Plants 2.

Langmead, B., Trapnell, C., Pop, M., and Salzberg, S.L. (2009). Ultrafast and memoryefficient alignment of short DNA sequences to the human genome. Genome Biol. 10: R25.

Law, J.A., Du, J., Hale, C.J., Feng, S., Krajewski, K., Palanca, A.M.S., Strahl, B.D., Patel, D.J., and Jacobsen, S.E. (2013). Polymerase IV occupancy at RNA-directed DNA methylation sites requires SHH1. Nature 498: 385-389.

Li, S., Vandivier, L.E., Tu, B., Gao, L., Won, S.Y., Li, S., Zheng, B., Gregory, B.D., and Chen, X. (2015). Detection of Pol IV/RDR2-dependent transcripts at the genomic scale in Arabidopsis reveals features and regulation of siRNA biogenesis. Genome Res. 25: $235-245$.

Lisch, D. (2013). How important are transposons for plant evolution? Nat. Rev. Genet. 14: 4961.

Liu, W., Duttke, S.H., Hetzel, J., Groth, M., Feng, S., Gallego-Bartolome, J., Zhong, Z., Kuo, H.Y., Wang, Z., Zhai, J., Chory, J., and Jacobsen, S.E. (2018). RNA-directed DNA methylation involves co-transcriptional small-RNA-guided slicing of polymerase $\mathrm{V}$ transcripts in Arabidopsis. Nat. Plants 4: 181-188.

Long, J., Walker, J., She, W., Aldridge, B., Gao, H., Deans, S., and Feng, X. (2021). Nurse cell-derived small RNAs define paternal epigenetic inheritance in Arabidopsis. bioRxiv.

Louvet, R., Cavel, E., Gutierrez, L., Guénin, S., Roger, D., Gillet, F., Guerineau, F., and Pelloux, J. (2006). Comprehensive expression profiling of the pectin methylesterase gene family during silique development in Arabidopsis thaliana. Planta 224: 782-791.

Love, M.I., Huber, W., and Anders, S. (2014). Moderated estimation of fold change and dispersion for RNA-seq data with DESeq2. Genome Biol. 15: 550.

Lu, J., Zhang, C., Baulcombe, D.C., and Chen, Z.J. (2012). Maternal siRNAs as regulators of parental genome imbalance and gene expression in endosperm of Arabidopsis seeds. 
Proc. Natl. Acad. Sci. U. S. A. 109: 5529-5534.

Lyons, E., Pedersen, B., Kane, J., Alam, M., Ming, R., Tang, H., Wang, X., Bowers, J., Paterson, A., Lisch, D., and Freeling, M. (2008). Finding and comparing syntenic regions among Arabidopsis and the outgroups papaya, poplar, and grape: CoGe with rosids. Plant Physiol. 148: 1772-1781.

Matzke, M.A. and Mosher, R.A. (2014). RNA-directed DNA methylation: an epigenetic pathway of increasing complexity. Nat. Rev. Genet. 15: 394-408.

Quinlan, A.R. and Hall, I.M. (2010). BEDTools: a flexible suite of utilities for comparing genomic features. Bioinformatics 26: 841-842.

Rodrigues, J.A., Ruan, R., Nishimura, T., Sharma, M.K., Sharma, R., Ronald, P.C., Fischer, R.L., and Zilberman, D. (2013). Imprinted expression of genes and small RNA is associated with localized hypomethylation of the maternal genome in rice endosperm. Proc. Natl. Acad. Sci. U. S. A. 110: 7934-7939.

Schmitz, R.J., He, Y., Valdés-López, O., Khan, S.M., Joshi, T., Urich, M.A., Nery, J.R., Diers, B., Xu, D., Stacey, G., and Ecker, J.R. (2013). Epigenome-wide inheritance of cytosine methylation variants in a recombinant inbred population. Genome Res. 23: $1663-1674$.

Singh, J., Mishra, V., Wang, F., Huang, H.-Y., and Pikaard, C.S. (2019). Reaction Mechanisms of Pol IV, RDR2, and DCL3 Drive RNA Channeling in the siRNA-Directed DNA Methylation Pathway. Mol. Cell 75: 576-589.e5.

Stroud, H., Greenberg, M.V.C., Feng, S., Bernatavichute, Y.V., and Jacobsen, S.E. (2013). Comprehensive analysis of silencing mutants reveals complex regulation of the Arabidopsis methylome. Cell 152: 352-364.

Tsuzuki, M., Sethuraman, S., Coke, A.N., Rothi, M.H., Boyle, A.P., and Wierzbicki, A.T. (2020). Broad noncoding transcription suggests genome surveillance by RNA polymerase V. Proc. Natl. Acad. Sci. U. S. A. 117: 30799-30804.

Wang, X. et al. (2011). The genome of the mesopolyploid crop species Brassica rapa. Nat. Genet. 43: 1035-1039.

Wei, W., Ba, Z., Gao, M., Wu, Y., Ma, Y., Amiard, S., White, C.I., Rendtlew Danielsen, J.M., Yang, Y.-G., and Qi, Y. (2012). A role for small RNAs in DNA double-strand break repair. Cell 149: 101-112.

White, J.L. and Kaper, J.M. (1989). A simple method for detection of viral satellite RNAs in small plant tissue samples. J. Virol. Methods 23: 83-93.

Wierzbicki, A.T., Ream, T.S., Haag, J.R., and Pikaard, C.S. (2009). RNA polymerase V transcription guides ARGONAUTE4 to chromatin. Nat. Genet. 41: 630-634. 
Williams, B.P., Pignatta, D., Henikoff, S., and Gehring, M. (2015). Methylation-sensitive expression of a DNA demethylase gene serves as an epigenetic rheostat. PLoS Genet. 11: e1005142.

Yang, L. and Bennetzen, J.L. (2009). Structure-based discovery and description of plant and animal Helitrons. Proc. Natl. Acad. Sci. U. S. A. 106: 12832-12837.

Zemach, A., Kim, M.Y., Hsieh, P.-H., Coleman-Derr, D., Eshed-Williams, L., Thao, K., Harmer, S.L., and Zilberman, D. (2013). The Arabidopsis nucleosome remodeler DDM1 allows DNA methyltransferases to access H1-containing heterochromatin. Cell 153: 193-205.

Zhai, J. et al. (2015). A One Precursor One siRNA Model for Pol IV-Dependent siRNA Biogenesis. Cell 163: 445-455.

Zheng, B., Wang, Z., Li, S., Yu, B., Liu, J.-Y., and Chen, X. (2009). Intergenic transcription by RNA polymerase II coordinates Pol IV and Pol V in siRNA-directed transcriptional gene silencing in Arabidopsis. Genes Dev. 23: 2850-2860.

Zhou, M., Coruh, C., Xu, G., Bourbousse, C., Lambolez, A., and Law, J.A. (2021). The CLASSY family controls tissue-specific DNA methylation patterns in Arabidopsis. bioRxiv.

Zhou, M., Palanca, A.M.S., and Law, J.A. (2018). Locus-specific control of the de novo DNA methylation pathway in Arabidopsis by the CLASSY family. Nat. Genet. 50: 865-873.

Zilberman, D., Gehring, M., Tran, R.K., Ballinger, T., and Henikoff, S. (2007). Genomewide analysis of Arabidopsis thaliana DNA methylation uncovers an interdependence between methylation and transcription. Nat. Genet. 39: 61-69.

Zou, C., Lehti-Shiu, M.D., Thibaud-Nissen, F., Prakash, T., Buell, C.R., and Shiu, S.-H. (2009). Evolutionary and expression signatures of pseudogenes in Arabidopsis and rice. Plant Physiol. 151: 3-15. 